\section{Dünnschichtchromatographische Trennung quaternärer Ammoniumverbindungen auf Celluloseschichten}

Im folgenden soll kurz ïber die dünnschichtchromatographische Trennung von aliphatischen, quaternären Ammoniumverbindungen berichtet werden, da diese Methode gegenüber der papierchromatographischen Auftrennung dieser Verbindungen einige Vorteile aufweist, von denen. vor allem die wesentlich kürzeren Laufzeiten und die grössere Nachweisempfindlichkeit hervorgehoben seien. Die Tatsache, dass auf den Dünnschichtchromatogrammen noch $0,5-1,0 \mu \mathrm{g}$ einer quaternären Ammoniumverbindung einwandfrei nachweisbar sind, ist vor allem für Untersuchungen iber das Vorkommen und den Stoffwechsel solcher Verbindungen in Pflanzen ${ }^{1-3}$ von nicht $\mathrm{zu}$ unterschätzender Bedeutung.

Als Sorptionsmittel wurde das von der Firma Macherey, Nagel \& Co., Düren, hergestellte normale Cellulosepulver, Sorte MN 300 ohne Gipszusatz, verwendet, das, völlig glatte, in den verwendeten Fliessmitteln ausgezeichnet

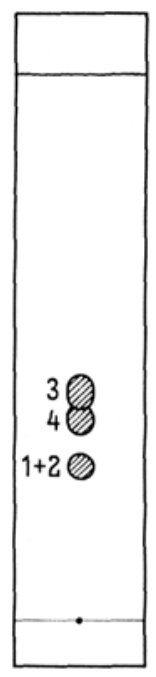

a

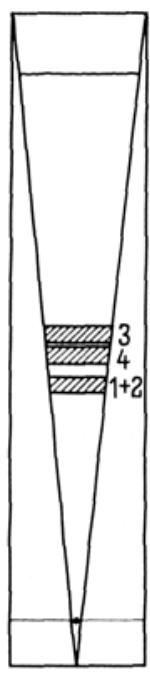

b

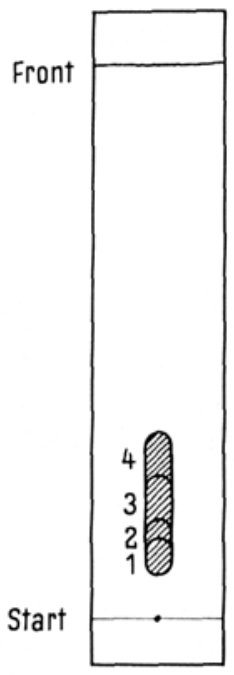

C

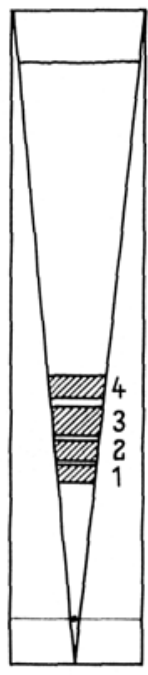

Dinnschich tchromatographische Trennung quaternärer Ammoniumverbindungen auf Celluloseschichten in $n$-Butanol-Eisessig-Wasser $4: 1: 5$ ( $a$ und $b$ ) und in Chloroform-Methanol-Wasser 75:22:3 (c und d). 1 Cholinchlorid, 2 Tetramethylammoniumchlorid, 3 Chlorcholinchlorid, 4- Acetylcholinchlorid. auf den Glasplatten haftende Schichten ergibt. Der Nachweis der quaternären Ammoniumverbindungen auf den Celluloseschichten wurde mit dem modifizierten Dragendorff-Reagens nach THIEs und Reurfer 4 durchgeführt. Dabei zeigen sich nach dem Besprühen deutliche Farbunterschiede zwischen den einzelnen Verbindungen (Tabelle). Von den verwendeten Fliessmitteln erwiesen sich das Gemisch n-Butanol-Eisessig-Wasser 4:1:5 und die Mischung Chloroform-Methanol-Wasser 75:22:3 als besonders geeignet. In Butanol-Eisessig-Wasser wandern aliphatische, quaternäre Ammoniumverbindungen in Form von gut abgegrenzten, runden Flecken, doch findet zwischen Cholinchlorid und Tetramethylammoniumchlorid keine Auftrennung statt (Figur, a). In Chloroform-Methanol-Wasser schieben sich die einzelnen Substanzen ineinander, lassen sich aber durch ihre unterschiedliche Anfärbung nach dem Besprühen mit dem Dragendorff-Reagens einwandfrei unterscheiden (Figur, c).

Eine wesentliche Verbesserung des Trenneffektes wurde durch Anwendung der Keilstreifentechnik erzielt ${ }^{5}$. Bei dieser Methode werden mit einer Metallspatel etwa $1 \mathrm{~mm}$ breite, V-förmige Abgrenzungslinien in die Celluloseschichten geritzt und das zu trennende Gemisch im schmalen Teil des so entstandenen Keiles punktförmig aufgetragen. Mit Hilfe dieser Technik gelingt in Chloroform-Methanol-Wasser die Auftrennung des Substanzgemisches in Form von scharf voneinander getrennten, schmalen Streifen (Figur, d). In Butanol-Eisessig-Wasser ist aber auch auf diese Art und Weise keine Trennung zwischen Cholinchlorid und Tetramethylammoniumchlorid zu erreichen (Figur, b).

Summary. Aliphatic, quaternary ammonium compounds can be separated by thin layer chromatography on cellulose layers. Application of the wedged-tip technique and use of the solvent system chloroform-methanolwater $75: 22: 3$ gave the best results.

\section{H. BAYZER}

Biologische Forschungsabteilung der Österreichischen Stickstoffwerke AG., Linz/Donau (Österreich), 28. Oktober 1963.

1 H. H. MAyr und E. PrEsoly, Planta 57,478 (1961).

8. H. Mayr und R. G. Paxton, Exper, 18, 440 (1962),

3 R. G. Paxton und H. H. Mayr, Planta 59, 165 (1962).

4 H. Thes und F. W. Reuther, Naturwiss. 41, 230 (1954).

- E. STAнL, Parfümerie und Kosmetik 39, 564 (1958).

\begin{tabular}{|c|c|c|c|}
\hline & \multicolumn{3}{|c|}{ Rst-Werte auf Keilstreifen } \\
\hline & $\begin{array}{l}\text { Farbreaktion mit } \\
\text { Dragendorff-Reagens }\end{array}$ & $\begin{array}{l}x \text {-Butanol-Eisessig } \\
\text { Wasser } 4: 1: 5\end{array}$ & $\begin{array}{l}\text { Chloroform-Methanol- } \\
\text { Wasser } 75: 22: 3\end{array}$ \\
\hline Tetramethylammoniumchlorid & braunrot & 1,00 & 1,17 \\
\hline $\begin{array}{l}\text { Cholinchlorid, } \\
\text { (2-Hydroxyathyl)-trimethylammoniumchlorid }\end{array}$ & violett & 1,00 & 1,00 \\
\hline $\begin{array}{l}\text { Chlorcholinchlorid, } \\
\text { (2-Chloräthyl)-trimethylammoniumchlorid }\end{array}$ & karminrot & 1,25 & 1,39 \\
\hline $\begin{array}{l}\text { Acetylcholinchlorid, } \\
\text { (2-Acetoxyäthyl)-trimethylammoniumchlorid }\end{array}$ & orangerot & 1,17 & 1,54 \\
\hline
\end{tabular}

s Anstelle von Rf-Werten werden die besser reproduzierbaren RSt-Werte, mit Cholinchlorid als Bezugssubstanz, angegeben:

$\mathrm{RSt}=\frac{\text { Entfernung des Analysensubstanzfleckes vom Start }}{\text { Entfernung des Cholinchloridfleckes vom Start }}$ 